\title{
Marges de l'emploi et protection sociale
}

Une analyse sociohistorique

Mathieu Grégoire et Odile Join-Lambert

\section{(2) OpenEdition}

\section{Journals}

Édition électronique

URL : http://journals.openedition.org/travailemploi/7371

DOI : 10.4000/travailemploi.7371

ISSN : 1775-416X

Éditeur

DARES - Ministère du Travail

Édition imprimée

Date de publication : 1 janvier 2017

Pagination : 5-16

ISSN : 0224-4365

Référence électronique

Mathieu Grégoire et Odile Join-Lambert, « Marges de l'emploi et protection sociale », Travail et Emploi [En ligne], 149 | janvier-mars 2017, mis en ligne le 11 juillet 2019, consulté le 24 septembre 2020. URL http://journals.openedition.org/travailemploi/7371 ; DOI : https://doi.org/10.4000/travailemploi.7371 


\title{
Introduction
}

\section{Marges de l'emploi et protection sociale Une analyse sociohistorique*}

\author{
Mathieu Grégoire $^{* *}$, Odile Join-Lambert ${ }^{* * *}$
}

$\mathrm{L}$

e système de protection sociale français s'est construit après la Seconde Guerre mondiale comme une architecture complexe et non comme un système unique de protection s'appliquant à l'ensemble de la population. Certaines catégories de travailleurs peuvent recourir à des formes de protection concurrentes au système général. Il est toutefois réputé reposer avant tout sur un modèle d'emploi standard (c'est-à-dire salarié, en contrat à durée indéterminée [CDI], à temps plein et intégré à une carrière complète et sans rupture) et souvent qualifié de pilier d'une «société salariale »(CASTEL, 1995). Corrélativement, il lui est reproché de mal, voire de ne pas ou peu couvrir ceux qui s'en écartent soit parce que leur emploi est précaire, soit parce qu'ils ne sont pas salariés, ou encore parce que leurs carrières sont marquées par une forte discontinuité. Dans ce contexte, l'avènement d'un chômage de masse et d'une dynamique de déstabilisation et de transformation de l'emploi suscite depuis une vingtaine d'années des interrogations sur ce système de protection sociale. Du rapport Boissonnat de 1995 sur Le Travail dans vingt ans (COMMISSARIAT GÉNÉRAL DU PLAN, 1995) aux débats actuels sur le renouveau de l'indépendance ou sur l' " ubérisation », en passant par l'idée défendue par Robert CASTEL (2007) de l'essor d'un « précariat » aux marges de l'emploi et de la protection sociale, le constat est sensiblement toujours de même nature : le système de protection sociale étant fondé sur un emploi standard, il serait aujourd'hui inadapté à de nouvelles formes d'emploi et de carrières beaucoup plus instables, discontinues et pas toujours (continûment) salariées. En particulier, selon cette perspective, le caractère « contributif » du système, c'est-à-dire le fait que les prestations sont proportionnelles aux contributions, engendrerait nécessairement

\footnotetext{
* Ce dossier fait suite à un appel à contribution. Catherine Omnès a participé à la rédaction de l'appel et à la sélection des articles reçus.

** Institutions et dynamiques historiques de l'économie et de la société (IDHES), université Paris-Nanterre ; mgregoire@parisnanterre.fr.

*** Professions, institutions, temporalités (Printemps), université de Versailles-Saint-Quentin-en-Yvelines ; odile.join-lambert@uvsq.fr.
} 
une marginalisation de tous ceux qui s'écartent de l'emploi standard : non seulement seuls ceux qui cotisent entrent dans le périmètre de couverture mais le niveau de leurs droits dépend étroitement de celui de leurs cotisations, donc des niveaux de salaire et de la durée d'emploi. En un mot, le modèle français de protection sociale serait particulièrement pénalisant pour tous ceux qui ne cotisent pas, ou pas assez, ou mal. En particulier, trois figures sociales (non exclusives les unes des autres) sont emblématiques d'une mauvaise articulation entre emploi et protection sociale : celle du salarié à l'emploi précaire (qu'il soit intérimaire, intermittent, saisonnier ou, plus banalement, qu'il alterne contrats à durée déterminée [CDD] et périodes de chômage), celle du travailleur non salarié (comme l'exploitant agricole ou l'artisan), celle du salarié à la carrière discontinue (dont le groupe des femmes est le plus significatif).

Les trois articles de ce dossier, quoique issus de disciplines et d'approches différentes, interrogent cette perspective associant étroitement une bonne couverture sociale à l'emploi standard en s'intéressant chacun à l'une ou à plusieurs de ces trois figures sur le temps long. L'article de Jean-Louis Escudier, intitulé «L'emploi et la protection sociale dans l'agriculture au filtre des rapports de genre. Les salariées agricoles aux XIX et $\mathrm{XX}^{\mathrm{e}}$ siècles », est consacré aux évolutions de la protection sociale et des conditions d'emploi des salariées agricoles. La figure du petit indépendant est au cœur de l'article de Marine Guichard et de Marielle Poussou-Plesse, «L'artisan, son comptable et sa retraite. Étude d'un profil d'indépendants établis » qui étudie les rapports complexes entretenus par les artisans à leur système de protection sociale (le régime social des indépendants [RSI]). Enfin, l'article de Karine Briard, « La retraite des salariés du secteur privé en France. Conjuguer redistribution, complexité et incertitude temporelle » constitue une analyse fine de l'histoire du régime de retraite vue à travers ses outils, permettant de comprendre comment les différents écarts au modèle de l'emploi standard peuvent être (ou non) considérés comme pénalisants en matière de retraite.

La première partie de cette introduction, consacrée à une brève présentation de l'évolution historique du modèle français de protection sociale (et en particulier du système de retraite), montre qu' on ne saurait la réduire à la thèse d'un double âge d'or associant étroitement emploi stable et bonne protection sociale puis d'une double période de crise de l'emploi standard et de la protection sociale. Au contraire, ce rapide rappel de la genèse du système de retraite en France entend montrer que la réalité de la protection sociale est multiforme et se laisse mal enfermer dans une caractérisation univoque, d'autant plus que ces dispositifs ont beaucoup évolué dans le temps. Jusqu'à quel point peut-on affirmer que le système de protection sociale protège mal, voire ne protège pas du tout, les profils d'emploi non standards ? Jusqu'à quel point cette caractéristique peut-elle être associée à la «nature » d'un système d'assurances sociales essentiellement favorable aux seuls salariés dont l'emploi est stable et les carrières continues plutôt qu'à ses transformations progressives ?

La seconde partie présente et fait discuter les trois articles du dossier qui se structurent autour de deux axes. Le premier consiste à privilégier une entrée par les 
dispositifs en interrogeant leur logique interne de financement et d'allocation des prestations afin de mesurer à quel point ils sont peu favorables à diverses marges de l'emploi standard. Le second s'intéresse aux formes d'emplois ou aux groupes sociaux qui les occupent. Cette optique nécessite ainsi d'étudier non seulement le degré de couverture de ces groupes sociaux aux marges de l'emploi standard, mais aussi les rapports souvent complexes qu'ils entretiennent à la protection sociale en général et à leur protection sociale en particulier. Dans les deux cas, ces articles éclairent les relations non univoques entre les marges de l'emploi et la protection sociale.

\section{Interroger le lien entre la construction de la protection sociale et l'évolution de l'emploi : l'exemple des retraites}

Au XIX ${ }^{\mathrm{e}}$ siècle, la protection sociale se réduit le plus souvent à l'alternative entre prévoyance individuelle pour ceux qui en sont capables et secours issus de l'assistance ou de la bienfaisance privée pour les « pauvres ». Mais déjà émergent en parallèle, le plus souvent à l'initiative des employeurs, des dispositifs de protection sociale liés à l'appartenance à une entreprise, à l'occupation d'un poste ou à un statut spécifique. Des réalisations fondées sur des logiques corporatistes ou paternalistes voient le jour, par exemple chez les mineurs ou les cheminots. Les fonctionnaires bénéficient dès 1853 d'une pension civile financée par répartition. À la fin du XIX ${ }^{\mathrm{e}}$ siècle, ce sont donc seulement certaines catégories qui bénéficient d'une couverture sociale et plus particulièrement d'une pension de vieillesse (marins, militaires, fonctionnaires civils, mineurs, cheminots) (ComitÉ D’hISTOIRE DE LA SÉcurité SoCIALE, TAURAN, 2005). Ces dispositifs concernent avant tout des personnels qualifiés et laissent de côté les plus mal rémunérés, dont la vieillesse est synonyme de misère. En fait, une grande partie des actifs meurt alors qu'elle travaille encore et dépend avant tout de sa propre capacité à s'auto-protéger dans des mécanismes de prévoyance individuelle pour les plus riches ou de mécanismes d'assistance publique ou de charité privée pour les plus pauvres.

Dans la première partie du $\mathrm{Xx}^{\mathrm{e}}$ siècle, la prévoyance individuelle et l'assistance laissent peu à peu la place à des mécanismes d'assurances sociales avec l'idée qui émerge d'une obligation de cotisation pour les salariés les plus modestes. Progressivement tous les indicateurs concordent : augmentation des âges moyen et médian de décès, de l'espérance de vie aux divers âges et du pourcentage des personnes âgées dans la population totale. Pourtant, en dehors de celles et ceux appartenant à des classes au sein desquelles les revenus sont suffisants pour constituer de façon substantielle et continue une épargne de prévision (FELLER, 2017), peu d'hommes et surtout de femmes peuvent compter sur une «pension de vieillesse ». C'est la raison pour laquelle un nombre important de personnes âgées, voire très âgées, doivent continuer à travailler (LAROQue, 1999). La perspective d'une «pension de vieillesse », de plus en plus souvent appelée retraite, correspond à une demande sociale croissante. Mais la réponse que les pouvoirs publics instaurent avec les retraites ouvrières et paysannes (ROP) 
de 1910, puis avec les lois de 1928 et de 1930 sur les assurances sociales, conforte un système à deux ou trois vitesses. Pour les catégories les plus aisées, il n'existe pas d'obligation de cotiser : la prévoyance individuelle demeure la norme. L'obligation de cotiser concerne uniquement les plus modestes. Et en pratique, cette obligation ne permet pas de couvrir l'intégralité de cette partie de la population. Dans les faits, par exemple, les ROP demeurent largement inappliquées. La couverture de la population féminine en âge de bénéficier d'une pension de retraite est extrêmement médiocre : 2,5 millions de salariées sont assujetties à titre obligatoire ; 2,1 millions de femmes sont par ailleurs couvertes en tant qu'épouses d'assujettis obligatoires. Au total, les femmes bénéficiant d'une pension représentent moins de la moitié du total de la population potentiellement concernée. Pour les 12 millions d'hommes en âge de bénéficier d'une pension de retraite, 7,5 millions environ perçoivent une pension (dont 5,4 à titre obligatoire), soit plus de $60 \%$ des Français et résidents étrangers de sexe masculin. Par ailleurs, beaucoup (en particulier les ouvriers) meurent avant l'âge de la retraite fixé à 65 ans (d'après le recensement de 1911, seule 8,4 \% de la population a plus de 65 ans) justifiant ainsi que la Confédération générale du travail (CGT) qualifie la ROP de retraite pour les morts (LAROQUE, 1999).

Le bilan des lois de 1928 et 1930 (qui intègrent les risques maladie et maternité, le risque vieillesse et le risque invalidité, dans l'ensemble plus vaste des assurances sociales, comprenant en outre une modeste protection contre le risque décès) n'est pas de nature différente. Là encore, l'architecture du dispositif, fondée sur l'obligation de cotiser pour les seuls travailleurs percevant un salaire inférieur à un plafond, écarte de fait la population la plus solvable des mécanismes de solidarité : les strates supérieures des salariés, les fonctionnaires mais aussi tous ceux qui ne sont pas salariés (indépendants, artisans, agriculteurs...) relèvent soit de la prévoyance individuelle, soit de dispositifs d'entreprises ou statutaires. Par ailleurs, le système couvre mal des franges non négligeables du salariat concernées par la flexibilité et l'intermittence de l'emploi (GrÉGOIRE, 2013).

La mise en place de la Sécurité sociale après la Libération a pu faire figure d'apogée de la dynamique de salarisation de la protection sociale en France. Les confédérations syndicales de salariés occupent une place dominante dans la gestion du dispositif jusqu' aux ordonnances de 1967, qui consacrent le retour du patronat dans une gestion paritaire. Financièrement, c'est une péréquation des cotisations à l'échelle interprofessionnelle qui entend permettre la mise en place d'une couverture homogène de l'ensemble des salariés et de leurs ayants droit. Mais le périmètre de la protection sociale ne s'arrête pas au seul salariat (BEC, 2014) : la loi du 22 mai 1946 pose le principe de la généralisation de la Sécurité sociale aux « personnes exerçant une activité professionnelle non salariée ». Pour ceux qui n'ont pas acquis de droits propres à la retraite, ou dont les cotisations ne permettent pas d'atteindre un montant minimum, l'État crée, en 1956, le Fonds national de solidarité et instaure le minimum vieillesse. Enfin, l'intégration des revenus les plus élevés passe par la mise en place de régimes complémentaires de retraite, d'abord pour les cadres en 1947 (Association 
générale des institutions de retraite complémentaire des cadres [Agirc]), puis pour les non-cadres en 1961 (Association pour le régime de retraite complémentaire des salariés [Arrco]). Néanmoins, les régimes spéciaux demeurent nombreux pour certains salariés bénéficiant de droits sociaux avancés (COMITÉ D'HISTOIRE DE LA SÉCuRITÉ SOCIALE, TAURAN, 2015). Quant aux autres actifs (indépendants [BRUNO, 2014], professions libérales...), ils accèdent progressivement à une protection sociale, mais dans des caisses spécifiques, marquant leur souhait de se tenir à distance de la solidarité interprofessionnelle de la Sécurité sociale.

\section{Marges de l'emploi et protection sociale : parcours de lecture des articles}

\section{L'entrée par les dispositifs : la contributivité, caractéristique inhérente au dispositif français ou dynamique récente?}

Avant d'aller plus loin dans l'analyse des dispositifs, il n'est pas inutile de s'arrêter un instant sur la notion de « contributivité » et sur ses usages parfois disparates. Dans un premier sens, le qualificatif de « contributif» sert à distinguer deux types de voies d'accès à la protection sociale : celles qui supposent une cotisation préalable et celles qui ne la supposent pas. Autrement dit, dans ce sens très large, il s'agit simplement de différencier, d'un côté, des assurances sociales financées par des cotisations sociales et, de l'autre, des dispositifs d'assistance financés par d'autres moyens, c'est-à-dire essentiellement par l'impôt. Dans cette perspective, il n'y a pas forcément lieu de proportionner strictement les prestations versées aux contributions : seul le fait d'avoir ou non à contribuer pour y être éligible suffit à déterminer le caractère contributif ou non du dispositif. Dans un second sens, plus strict, le qualificatif de « contributif » sert à désigner le degré de correspondance, pour chaque bénéficiaire, entre les prestations qu'il reçoit et les cotisations qu'il a préalablement versées. Autrement dit, il s'agit de mesurer à quel point un dispositif relève d'une logique de salaire différé (fonctionnant ainsi, pour chaque individu, comme une forme d'épargne obligatoire).

Cette distinction n'est pas sans incidence sur le sort réservé aux salariés à l'emploi atypique ou aux carrières discontinues. Un dispositif dit « contributif » au premier sens (en ce qu'il exige une contribution minimale par exemple) peut très bien être faiblement contributif dans le second sens, en ne proportionnant pas de manière stricte le niveau de prestation à la quantité d'emploi. Ainsi, par exemple, la durée d'un congé maternité n'est pas proportionnée à la durée de cotisation comme peuvent l'être les indemnités de chômage : il n'est pas contributif dans le second sens. Néanmoins, le dispositif des congés maternité est contributif dans le premier sens car l'éligibilité aux indemnités journalières y est conditionnée à un minimum de 150 heures d'emploi par trimestre, ce qui le rend accessible à une grande partie des salariées à l'emploi précaire qui en bénéficient de façon identique aux salariées à l'emploi stable. 
On peut se demander si la confusion entre ces deux sens du terme de « contributivité » n'est pas source d'un certain nombre d'anachronismes. En effet, la contributivité stricte, au sens d'un idéal de proportion entre cotisations et prestations, voire de «neutralité actuarielle », correspond à une dynamique et à un usage récents des réformes des systèmes de protection sociale vers davantage de contributivité. Dès lors, n'est-ce pas excessif de considérer que cette exigence prévalait dès l'origine de ces systèmes de protection sociale ? Par exemple, lors de la mise en place en 1947 du régime de retraite complémentaire des cadres, l'Agirc, système contributif s'il en est puisqu'il fonctionne par "points », le calcul des droits à la retraite s'est opéré sans référence à des cotisations préalables, mais sur la base de reconstitutions de carrières.

L'évolution de l'assurance chômage marque une dynamique de glissement du premier sens large de la contributivité à son second sens strict. En 1979, la convention d'assurance chômage prévoyait qu'étaient éligibles tous les salariés ayant contribué au moins trois mois durant les douze derniers mois (DANIEL, TUCHZSIRER, 1998). Autrement dit, ce critère visait surtout à distinguer les salariés (précaires ou pas) de non salariés qui ne cotisaient pas. Mais les droits acquis par les uns et les autres étaient identiques : douze mois complets d'indemnisation. L'évolution du dispositif a été depuis marquée par une mise en relation de plus en plus étroite des montants cotisés et des prestations versables : d'abord par la mise en place de filières d'indemnisation dépendant des durées de cotisation en 1984 puis, plus récemment, par la mise en œuvre du principe « un jour cotisé, un jour indemnisé » en 2009 et des droits rechargeables en 2014. En d'autres termes, l'assurance chômage n'est pas d'emblée et par nature associée à une forte contributivité, elle l'est progressivement devenue.

Le même type d'évolution complexe peut être identifié pour le régime de retraite. C'est ce que montre le premier article de ce dossier qui est consacré à l'analyse fine du dispositif et à l'étude de ses implications en termes d'allocation des ressources sur soixante-dix ans. K. Briard y analyse, en économiste, l'évolution des droits pour les salariés les plus susceptibles de s'écarter du modèle de l'emploi standard et des carrières continues. Le premier apport de cet article est ainsi de rappeler que le régime général de retraite a toujours eu une dimension non contributive (que l'auteure appelle « redistributive »). De nombreux mécanismes assurent depuis longtemps cette fonction « non contributive » qui permet une couverture large des salariés, y compris lorsqu'ils ont des emplois précaires et des carrières discontinues. Tous les salaires ne sont pas pris en compte dans le calcul du montant de la pension, mais seulement les meilleurs. Par ailleurs, les conditions de validation des trimestres requis pour être éligible à la retraite ne correspondent pas à la durée travaillée, mais à des seuils de rémunérations relativement accessibles y compris pour des salariés à l'emploi très discontinu.

Le second intérêt de l'article de K. Briard est de montrer que les évolutions du régime de retraite sont ambiguës : certaines vont dans un sens plus favorable à la couverture des parcours les plus discontinus, d'autres dans un sens moins favorable. Sans entrer dans le détail des analyses, prenons seulement un exemple frappant : celui du critère de carrière complète exigé pour l'éligibilité à la pension. À partir des 
années 1970, des trimestres sans cotisations peuvent aussi être validés pour couvrir des situations de chômage, de maladie, de service national tandis que dans le même temps, le régime va vers davantage de contributivité. Alors que la condition de durée était à l'origine de seulement 15 ans pour faire valoir son droit à retraite à 65 ans, une condition de carrière complète à 37,5 annuités est exigée à partir de 1971 et ira en s'accentuant jusqu'à 43 annuités pour les personnes nées après 1973. On mesure, en particulier pour les carrières discontinues des femmes, à quel point une telle évolution de la législation (et non la « nature » du dispositif mis en place en 1945) contribue à rendre difficile l'accès à une retraite à taux plein pour les femmes qui ont interrompu leur carrière pour élever des enfants.

Parmi les dispositions qui permettent le plus de s'écarter d'une logique purement contributive, $\mathrm{K}$. Briard insiste sur la pension minimum, la validation de trimestres au chômage, ou encore, les droits liés à la famille (validation de trimestres et bonifications pour enfant). Leurs effets sont le plus souvent positifs pour ceux dont les carrières sont les moins continues et/ou les moins bien rémunérées. Mais ce n'est pas toujours nécessairement le cas et certains dispositifs non contributifs aboutissent à des effets anti-redistributifs ou ambigus.

Ces évolutions contradictoires - faites d'accentuation de la contributivité et de multiplication de mesures correctives aux effets incertains - impliquent que les liens entre marché du travail et système de retraites sont particulièrement complexes et, en tout cas, non univoques. Dans un contexte de montée en puissance des parcours atypiques et face à la complexité d'un système dont les effets sont parfois non maîtrisés et inattendus, l'un des enjeux politiques forts est ainsi, selon l'auteure, d'approfondir les capacités d'expertise et d'évaluation des anciennes et futures réformes.

\section{L'entrée par les formes d'emploi et par les groupes sociaux : entre défiance, relégation et réappropriation}

Une deuxième entrée possible pour questionner le lien entre formes non standards d'emplois et protection sociale consiste, à partir des groupes sociaux eux-mêmes et de leurs formes d'emplois spécifiques, à s'interroger d'une part sur leur couverture par les dispositifs de protection sociale, d'autre part sur les rapports qu'ils entretiennent à l'égard de cette protection sociale.

Là aussi, l'analyse du lien entre emploi atypique et protection sociale s'avère complexe et non réductible à une simple relégation subie par ces salariés. Certes, ces effets de relégation ont bien sûr existé. Le cas des salariés intermittents du spectacle en est un exemple. La mise en place des assurances sociales en 1928 et 1930, ou celle de la Sécurité sociale en 1945, conduisirent incontestablement à mettre en porte-à-faux leur emploi très intermittent avec les mécanismes de cotisations : une partie de leurs rémunérations très concentrées sur des durées d'emploi faibles dépassait les plafonds et ne leur ouvrait aucun droit alors que des journées chômées, sans aucun revenu, ne leur ouvraient pas davantage de droits (GRÉGOIRE, 2013 ; CARDON, 2014). 
Mais ces mécanismes de relégation ont fréquemment suscité des réactions qui peuvent déboucher sur une protection sociale propre à travers la constitution de régimes spéciaux ou, inversement, sur la revendication d'une adaptation du régime général aux spécificités de l'emploi de tel ou tel groupe. C'est ainsi qu'ont été créées dans les années 1960 l'ensemble des « annexes » au règlement de l'assurance chômage : les annexes 8 et 10 des artistes et techniciens du cinéma, de l'audiovisuel et du spectacle vivant en sont certainement l'exemple le plus emblématique, mais des « annexes » spécifiques destinées à adapter les règles à des spécificités d'emploi existent aussi pour les voyageurs, représentants et placiers (VRP), journalistes, personnels navigants de l'aviation civile, assistants maternels et assistants familiaux, bûcherons-tâcherons et agents rémunérés à la commission (annexe 1), les personnels navigants de la marine marchande, marins-pêcheurs (annexe 2), les ouvriers dockers (annexe 3), les intérimaires (annexe 4), les travailleurs à domicile (annexe 5), les salariés occupés expatriés (annexe 9), ou encore les apprentis et titulaires d'un contrat de professionnalisation (annexe 11).

Par ailleurs, la marginalisation de certains groupes aux caractéristiques d'emploi spécifiques peut relever non d'une dynamique de relégation subie, mais bien d'une résistance tout à fait assumée de ces groupes à leur intégration dans des mécanismes de solidarité large. De ce point de vue, les contributions à ce dossier de J.-L. Escudier d'une part, et de M. Guichard et M. Poussou-Plesse d'autre part, ont l'intérêt majeur de porter le regard sur deux zones sociales dans lesquelles se sont historiquement exprimées les plus fortes réticences, pour ne pas dire les plus fortes résistances, à l'idée même de sécurité sociale : les agriculteurs d'une part, les petits indépendants d'autre part. Comme le rappelle l'ouvrage pionnier d'Henri HATZFELD (1971) consacré aux origines de la Sécurité sociale, les principales résistances à la mise en place d'un étage " socialisé » du salaire visant à assurer une protection sociale aux travailleurs ne sont pas venues du grand patronat. Bien au contraire, c'est la défense de la petite propriété, dans une France dans laquelle agriculteurs et indépendants sont encore des forces sociales centrales, qui a constitué le frein essentiel à la mise en place des assurances sociales dans la première moitié $\mathrm{du} \mathrm{Xx} \mathrm{x}^{\mathrm{e}}$ siècle. Et le cœur de cette opposition résidait moins, pour ces groupes, dans le constat d'une inadaptation fonctionnelle des assurances sociales à leurs modalités d'emploi que dans une idéologie ou dans des représentations d'eux-mêmes fondamentalement incompatibles avec la socialisation inhérente à un système d'assurance sociale. Comme l'écrit H. HATZFelD (p. 302),

« les classes moyennes non salariées [...] se sont battues non pour un objectif limité mais pour un enjeu considérable : une certaine conception de l'homme dans la société, à laquelle leurs intérêts se trouvaient liés. Il s'agissait pour elles de défendre un type de société dont la petite propriété était l'élément constitutif essentiel. Or cette conception était inconciliable avec celle qui se dégageait de la législation sociale qu'on entendait bâtir. »

L'article de M. Guichard et de M. Poussou-Plesse permet de mesurer combien les résistances de la première moitié $\mathrm{du} \mathrm{Xx}^{\mathrm{e}}$ siècle à l'idée même de mutualisation analysée 
par H. HATZFELD demeurent aujourd'hui encore vivaces chez des petits indépendants. Leur contribution est en effet consacrée aux rapports qu'entretiennent des artisans du bâtiment au RSI et, plus particulièrement, à leur régime de retraite après un processus historique qui a globalement conduit, malgré ces résistances, à un « alignement » des régimes autonomes des non-salariés sur le régime général. En s'intéressant, en sociologues, aux «calculs économiques ordinaires » des artisans du bâtiment ainsi qu'aux conseils que leur délivrent leurs experts-comptables, M. Guichard et M. Poussou-Plesse montrent combien le consentement à cotiser demeure problématique pour ces franges de la population. Et cette question est d'autant plus prégnante que, contrairement aux salariés et malgré le caractère obligatoire de la cotisation, il demeure des marges de liberté dans le choix de l'assiette soumise à cotisation, dans celui de la forme juridique d'exercice ou encore dans la possibilité de soustraire une partie des revenus à la déclaration en travaillant « au noir». Il en ressort que, le régime fût-il en répartition, c'est en termes de rendement que ces choix sont opérés, en comparaison avec des investissements en capital. La stratégie d'optimisation à l'œuvre consiste ainsi à « cotiser au plafond » (p. 61), ni plus ni moins. Au-delà, les placements « dans la pierre » correspondent aux incitations à l'auto-protection sociale liée à la loi Madelin de 1994 qui accorde une défiscalisation spécifique aux indépendants. Mais ils correspondent encore à cet idéal de sécurité-propriété de la «maison aux volets verts » analysé par H. HATZFELD.

L'article de J.-L. Escudier est consacré aux évolutions de la protection sociale et des conditions d'emploi des salariées agricoles. L'intérêt de prendre pour objet celles qu'on peut historiquement identifier comme des « journalières » ou comme des «servantes de fermes » est multiple. En effet, l'article de J.-L. Escudier permet de porter l'attention sur un monde social qui se situe à l'intersection de plusieurs problématiques : celle d'un monde agricole dont on a déjà souligné sa défiance historique vis-à-vis de la construction d'une Sécurité sociale ; celle d'un emploi salarié particulièrement intermittent ; celle, enfin, d'une condition de femme (et d'épouse et de mère de famille) qui a fait l'objet d'un traitement spécifique à la fois par la protection sociale et par le droit du travail.

Appartenant au monde agricole, ces salariées subissent la défiance des agriculteurs (leurs employeurs) vis-à-vis de la construction des assurances sociales. J.-L. Escudier évoque ainsi par exemple le mouvement qui conduit ces agriculteurs, derrière le leader politique et syndical d'extrême droite Henri Dorgères et ses « chemises vertes », à ne pas honorer leur obligation légale de cotiser pour leurs salariés en 1935. C'est encore cette exception agricole qui conduit le législateur à justifier, jusqu'à la fin des années 1960, la fixation d'un salaire minimum (le salaire minimum agricole garanti [Smag]) inférieur au Smig (salaire minimum interprofessionnel garanti) mis en place en 1950.

En tant que salariées à l'emploi précaire, les salariées agricoles se trouvent - à l'instar des salariés du spectacle évoqués plus haut - en porte-à-faux avec les modalités de constitution des droits, que ce soit dans le cadre des retraites ouvrières et paysannes (ROP) à partir de 1910 ou dans celui des assurances sociales à partir de 
1935 (date du décret déterminant le régime des assurances sociales applicables aux salariés agricoles). Malgré leur affiliation, le caractère occasionnel de leur emploi les pénalise fortement.

Enfin, ce sont surtout les enjeux liés au caractère féminin de ces salariées qui retiennent l'attention. Soulignons d'abord l'importance des inégalités de genre dans la constitution des conditions d'emploi et des conditions de protection sociale. Ainsi au XIX ${ }^{e}$ siècle s'opère globalement, selon J.-L. Escudier, un partage sexué, entre les hommes qui sont surreprésentés dans le mouvement mutualiste et les femmes qui le sont dans les dispositifs d'assistance comme, par exemple, l'Assistance médicale gratuite mise en place en 1893. Le droit du travail n'est pas en reste : ainsi la loi de 1950 sur les conventions collectives autorise de fait que le sexe soit un critère retenu pour différencier les salaires conventionnels minima. Les conventions collectives agricoles de Côte-d'Or et du Var analysées par l'auteur distinguent ainsi explicitement un salaire masculin et un salaire féminin. Au-delà du caractère sexué de l'emploi ou de la protection sociale, c'est la rétroaction entre l'emploi et la protection sociale de ces salariées agricoles qui pose avec le plus d'acuité les enjeux croisés entre ces deux domaines. Le système de protection sociale peut en effet avoir en retour des effets sur l'emploi, voire sur l'identité des groupes sociaux qui les occupent. La constitution du groupe des « cadres » et le rôle qu'a joué la mise en place de l'Agirc en sont un exemple canonique (BOLTANSKI, 1982 ; FRIOT, 1998). Dans le cas des salariées agricoles, ce sont des dispositifs spécifiquement destinés aux familles (à travers les allocations familiales) ou visant spécifiquement les femmes au foyer (avec l' « allocation de salaire unique ») qui jouent un rôle déterminant sur leur emploi, en incitant massivement ces salariées à l'emploi occasionnel au retrait du marché du travail ou au travail au noir, en confortant leur rôle d'épouse et de mère.

Le cas des femmes illustre ainsi fort bien toute la complexité des liens entre protection sociale et emploi atypique. D'un côté, les dimensions contributives du système français jouent en défaveur des femmes dont les carrières sont discontinues et l'emploi plus souvent atypique (ne serait-ce qu'avec la plus forte prégnance des temps partiels) comme cela a été observé dans le cas des retraites (APROBERTS, CASTEL, 2016). En même temps, les mesures spécifiques dont le but est de corriger les effets de la contributivité prennent le risque en retour d'enfermer les bénéficiaires dans la situation qu'elles sont supposées compenser.

Au-delà de ces quelques éléments transversaux, chacun des trois articles souligne les inégalités de retraite entre hommes et femmes, et entre groupes sociaux, face aux carrières discontinues. La reprise dans le débat public de thèmes comme l'âge du départ en retraite ou la pénibilité ne suffit pas à éclairer les conditions de mise en œuvre des modèles de projection économiques et statistiques depuis les années 1950, leur portée et leurs incidences différenciées en fonction des périodes, du sexe et du groupe 
social des bénéficiaires (BLANCHET, LE GALLO, 2013). D'hier à aujourd'hui, quelles tentatives ont existé de prendre en compte dans ces modèles les inégalités à des fins de redistribution entre groupes sociaux et entre hommes et femmes ? Approfondir cette perspective de long terme, sur laquelle s'est engagé ce dossier, permettrait de mieux saisir les dispositifs dans le passé et de les interpréter en fonction des usages qu'en font les acteurs à chaque période, en fonction du marché du travail et de ses marges.

\section{BIBLIOGRAPHIE}

BEC C. (2014), La Sécurité sociale, une institution de la démocratie, Paris, Gallimard.

Blanchet D., Le Gallo F. (2013), « Baby-boom et allongement de la durée de vie : quelles contributions au vieillissement ? », Insee analyses, $\mathrm{n}^{\circ} 12$.

Boltanski L. (1982), Les Cadres : la formation d'un groupe social, Paris, Éditions de Minuit.

BRUNo A.-S. (2014), « Retour sur un siècle de protection sociale des travailleurs indépendants. France $\mathrm{XX}^{\mathrm{e}}$ siècle » in Célérier S. (dir.), Le Travail indépendant. Statut, activités et santé, Rueil-Malmaison, Éditions Liaisons, pp. 31-52.

CARDON V. (2014), Emploi, vieillissement et retraite dans les métiers des spectacles, rapport pour le ministère de la Culture et de la Communication, mars.

CAstel R. (1995), Les Métamorphoses de la question sociale. Une chronique du salariat, Paris, Fayard.

CASTEL R. (2007), « Au-delà du salariat ou en deçà de l'emploi ? L'institutionnalisation du précariat ? », in Paugam S. (dir.), Repenser la solidarité : l'apport des sciences sociales, Paris, Presses universitaires de France, pp. 415-433.

Comité D'histoire de la Sécurité sociale, TAURAN T. (coord.) (2015), La Sécurité sociale, son histoire à travers les textes, tome VII, Les Régimes spéciaux de Sécurité sociale, Paris, Association pour l'étude de l'histoire de la Sécurité sociale.

Commissariat général du Plan, Boissonnat J. (pr.), Mabit R. (rapp.) (1995), Le Travail dans vingt ans, Paris, Odile Jacob/La Documentation française.

DANiEl C., TuchsZiRER C. (1998), L'État face aux chômeurs. L'indemnisation du chômage de 1884 à nos jours, Paris, Flammarion.

Feller É. (2017), Du vieillard au retraité. La construction de la vieillesse dans la France du $X X^{e}$ siècle, $2^{\mathrm{e}}$ éd. Paris, L'Harmattan.

FRIOT B. (1998), Puissances du salariat : emploi et protection sociale à la française, Paris, La Dispute.

GRÉGOIRE M. (2013), Les Intermittents du spectacle. Enjeux d'un siècle de luttes (de 1919 à nos jours), Paris, La Dispute.

HatzFeld H. (1971), Du paupérisme à la Sécurité sociale. Essai sur les origines de la Sécurité sociale en France, 1850-1940, Paris, Armand Colin. 
LAROQUE M. (dir.) (1999), Contribution à l'histoire financière de la Sécurité sociale, Paris, La Documentation française, Association pour l'étude de l'histoire de la Sécurité sociale.

APRoberts L., CAStel N. (2016), « Les effets négatifs de la contributivité sur la retraite des femmes. Analyse et alternatives », Retraite et société, n ${ }^{\circ} 74$, pp. 165-183. 